\title{
DYNAMIC RANGE OF HARMONIC PHASE MAGNETIC RESONANCE IMAGING (HARP-MRI)
}

\author{
Vijay Parthasarathy ${ }^{1}$, Moriel Ness-Aiver ${ }^{2}$, and Jerry L.Prince ${ }^{1}$ \\ ${ }^{1}$ Electrical and Computer Engineering, Johns Hopkins University, Baltimore, MD \\ ${ }^{2}$ Diagnostic Radiology, University of Maryland School of Medical School, Baltimore, MD
}

\begin{abstract}
Harmonic phase (HARP) magnetic resonance imaging (MRI) is an image analysis method for measuring tissue motion and strain from tagged MR images. Previous studies in cardiac tagged MRI have demonstrated its utility in the characterization of myocardial infarction, ischemia, and the reperfusion of stunned myocardium. Recently HARP has also been used in characterizing the motion of the tongue during speech. In this paper, we characterize dynamic range — limits of elongation, compression and rotation — of HARPMRI. Viewing HARP-MRI as a phase demodulation technique, a key simplifying relationship permits us to derive approximate analytic expressions for these quantities. Simulations demonstrate the validity of our analysis and also help to provide intuition for these results.
\end{abstract}

\section{INTRODUCTION}

The harmonic phase (HARP) technique was introduced as a computational approach for the automated analysis of tagged magnetic resonance (MR) images [1] and has recently been shown to permit real-time imaging of myocardial motion and strain [2]. HARP has been used in the imaging of myocardial infarction and ischemia [3], in the evaluation of reperfusion of stunned myocardium [4], and in the rapid detection of ischemia in MR stress tests [5]. HARP has also been used in estimating the motion of the tongue during speech and swallowing [6]. It is important to understand the performance limitations of HARP in these various applications, both to explain anomalous behavior and to optimize imaging and processing parameters.

In this paper, we develop quantitative expressions for the dynamic range of HARP analysis in the regional estimation of elongation, compression, and rotation. Our approach exploits an analogy between HARP and modulation theory in communications. In particular, we interpret the instantaneous frequencies in tagged images in terms of Fourier frequencies in the spectra of the tagged images. Although this interpretation is valid only for small subset of images [7],

This research was supported by NIH/NHLBI grant R01 HL47405.



(a)

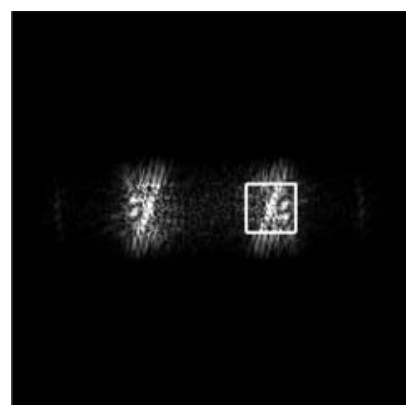

(b)
Fig. 1: (a) Tagged MR image and (b) its Fourier magnitude spectrum with bandpass filter around the harmonic peak. Both images are scaled and cropped for clarity.

we will see that this interpretation is valid for tagged images under consideration. This relation leads readily to analytic expressions that are both intuitively appealing and accurate in the simulations we have conducted.

\section{OVERVIEW OF HARP}

Fig. 1 shows a sinusoidally tagged MR image and its spectrum. The tag pattern is a zero-mean sinusoid [8], which yields only two spectral peaks in the frequency domain due to amplitude modulation caused by tag application [9]. A harmonic image can be computed by bandpass filtering one of the harmonic peaks, as shown in Fig. 1(b), and taking its inverse Fourier Transform. The resulting complex harmonic image can be expressed as [1]

$$
I(\mathbf{x}, t)=D(\mathbf{x}, t) e^{j \phi(\mathbf{x}, t)},
$$

where $\mathbf{x}=\left[\begin{array}{ll}x & y\end{array}\right]^{\mathrm{T}}$ represents the position on the image and $t$ the time interval after the application of the tags. The magnitude image $D(\mathbf{x}, t)$ is proportional to the transverse magnetization at position $\mathbf{x}$ and time $t$. The harmonic phase $\phi(\mathbf{x}, t)$ is related to tissue displacement $\mathbf{u}(\mathbf{x}, t)$ as follows.

$$
\phi(\mathbf{x}, t)=-\boldsymbol{\omega}_{0}^{\mathrm{T}} \mathbf{u}(\mathbf{x}, t)+\boldsymbol{\omega}_{0}^{\mathrm{T}} \mathbf{x},
$$

where $\boldsymbol{\omega}_{0}$ gives the orientation and frequency of the tag pattern in the image plane. The harmonic phase image there- 
fore contains information about the displacement of the tissue.

\section{COMMUNICATIONS VIEWPOINT}

We start by interpreting the underlying sinusoidal tag pattern as a carrier signal. It is apparent from Fig.1(a) and Eq. (1) that application of a tag pattern to a given image slice is an amplitude modulation of the tag pattern by the anatomy $D(\mathbf{x}, t)$. From Eq. (2), we see that the displacement $\mathbf{u}(\mathbf{x}, t)$ causes a phase modulation of the underlying tag pattern, where the phase modulation index is $-\boldsymbol{\omega}_{0}^{\mathrm{T}}$. The Eulerian strain is the spatial derivative of displacement; therefore, Eulerian strain can be interpreted as the "signal" that frequency modulates the carrier. Accordingly, the harmonic image $I(\mathbf{x}, t)$ is an AM-FM signal whose instantaneous amplitude is $D(\mathbf{x}, t)$ and whose instantaneous frequency (IF) is Eulerian strain.

Given this interpretation, HARP analysis can be viewed as a phase or frequency demodulation technique, and the estimation of Eulerian strain is essentially the estimation of the IF of an AM-FM image. In HARP the IF is estimated by taking derivative of the phase of the harmonic image, which is similar to the analytic signal used in the AM-FM signal analysis $[10,11]$. Even though the term instantaneous frequency' leads us to think of it as frequency of a local sinusoid at a particular position, IF and Fourier frequencies are fundamentally different quantities [12]. IF and Fourier frequencies not simply related except for a small subset of AM-FM signals viz. 'monocomponent' signals [7, 13, 14, $15]$.

In this paper, we use the fact that a tagged image with one tag direction is a monocomponent signal. We see the IF as the frequency of a sinusoid that locally (in space) fits the signal under analysis [13]. Even though we need tags in two directions to estimate strain in 2-D, the bandpass filtering and the subsequent estimation of IF is done separately for each direction and later combined. Hence the approximation of a monocomponent signal still holds. In this paper all the analysis is done for vertical tags and it extends to the horizontal tags in a straightforward way.

\section{DYNAMIC RANGE}

Using the above viewpoint we develop a simple theory for the dynamic ranges of elongation, compression, and rotation of tags. In HARP, we extract one harmonic peak using a bandpass filter $\mathrm{B}\left(k_{x}, k_{y}\right)$ centered around $\left[\begin{array}{ll}\omega_{0} & 0\end{array}\right]^{\mathrm{T}}$ in k-space (Fig. 2). The position and the shape the filter is determined by the tag frequency and the magnitude of motion [16]. In this discussion we assume a square filter [2] centered around $\left[\begin{array}{ll}\omega_{0} & 0\end{array}\right]^{\mathrm{T}}$ and the $\mathrm{N} \Delta k$ on the each side,

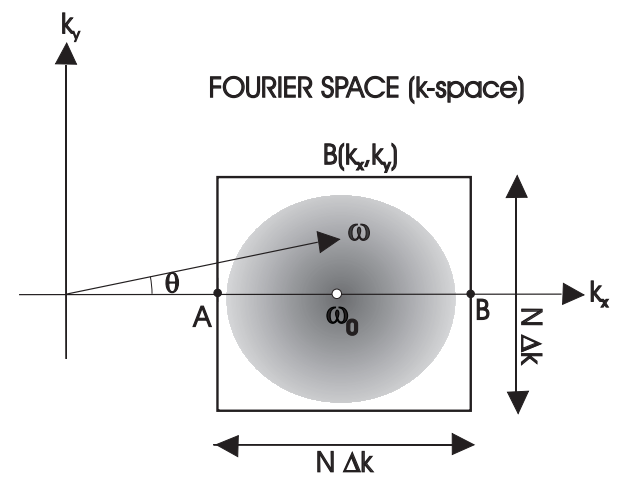

Fig. 2: Schematic of the bandpass filter in frequency domain

where $\mathrm{N}$ and $\Delta k$ are the number of samples and sampling interval (in $\mathrm{mm}^{-1}$ ) respectively.

The premise of HARP is that the IF of a tagged image is estimated by calculating the spatial gradient of the harmonic phase $\phi(\mathbf{x}, t)$. The spatial gradient $\nabla \phi(\mathbf{x}, t)$ defines a vector field of instantaneous frequencies. Viewing these IF vectors in light of the theory mentioned in Section 3, these vectors represent frequency of local sinusoids.

Let an IF vector be $\boldsymbol{\omega}(\mathbf{x}, t)=\omega(\mathbf{x}, t) \angle \theta(\mathbf{x}, t)$. The spectrum of an eternal sinusoid with frequency IF contains a impulse at $\boldsymbol{\omega}$ in frequency domain. So for each IF in the spatial domain, there is impulse associated with it in frequency space. Even though these sinusoids are not eternal, we use an approximation here. We claim that HARP can detect a tag pattern at $\mathbf{x}$ if the impulse in frequency (defined by IF at $\mathbf{x})$ remains within the bandpass filter. In other words, there is a certain dynamic range of motion that can be imaged by HARP which is determined by the size and position of the box. Mathematically, this claim can be written down by placing simple bounds on the $x$ and $y$ components of the IF.

Let $W=2 \pi \mathrm{N} \Delta k$ be size of the bandpass filter in rads $/ \mathrm{mm}$. Let $A=\omega_{0}-\frac{W}{2}$ and $B=\omega_{0}+\frac{W}{2}$, be the bounds of the filter along the $k_{x}$ axis (Fig. 2). Given $W, A$ and $B$ the IF $\omega \angle \theta$ will be perceived by HARP if the $x$ and $y$ components are fall within the horizontal and vertical extents of the filter i.e.

$$
\begin{aligned}
& A \leq \omega|\cos \theta| \leq B, \\
& 0 \leq \omega|\sin \theta| \leq \frac{W}{2} .
\end{aligned}
$$

The maximum and minimum value of $\omega$ is the dynamic range of compression and elongation respectively. The dynamic range of $\theta$ determines the dynamic range of the rotation of tags. The dynamic range of $\theta$ also determines the maximum tag bending HARP can see.

Equations 3 and 4 are coupled inequalities i.e. the dynamic range of elongation and compression is a function of $\theta$ and vice versa. By decoupling the inequalities and plotting them on $\omega-\theta$ plane (or equivalently strain-rotation 


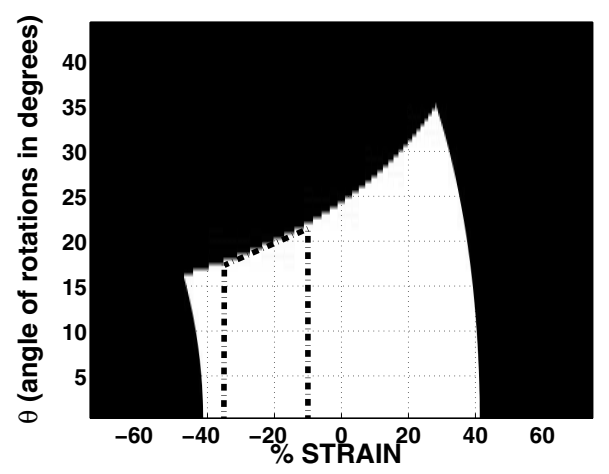

Fig. 3: Feasible region in strain-rotation space. White regions represent IFs that are within the dynamic range of HARP

space), we can visualize the dynamic ranges of elongation, compression and rotation for a given tagging and imaging parameters. By simple manipulations we can decouple eqs. 3 and 4 . The decoupled inequalities are given as follows.

$$
\begin{aligned}
& \max \left[\frac{A}{|\cos \theta|}, 0\right] \leq \omega \leq \min \left[\frac{B}{|\cos \theta|}, \frac{W}{2|\sin \theta|}\right], \\
& \cos ^{-1} \sqrt{\max \left[1-\frac{W^{2}}{4 \omega^{2}}, \frac{A^{2}}{\omega^{2}}\right]} \leq \theta \leq \cos ^{-1} \sqrt{\min \left[1, \frac{B^{2}}{\omega^{2}}\right]} \text {. }
\end{aligned}
$$

\section{RESULTS AND DISCUSSION}

Feasible region in "strain-rotation" space Consider the following tagging and imaging parameters: tag separation $7 \mathrm{~mm}$, FOV $280 \mathrm{~mm}$, and a bandpass filter with 33 samples on each side centered about tag frequency(used in [2]). Further assume Nyquist sampling of the image; $\Delta k=1 / \mathrm{FOV}$. These parameters will be consistently used throughout this section. The dynamic ranges can be visualized in feasible region images (Fig. 3) in strain-rotation space. The feasible region is calculated using the decoupled inequalities.

Now let's interpret Fig. 3 in terms of cardiac imaging. With the above parameters, the dynamic range of strain of $\pm 41.25 \%$ (negative is compression, positive is expansion) and maximum rotational angle range from $16^{\circ}-34^{\circ}$ degrees depending of the magnitude of strain. These ranges are quite ample for both normal and diseased hearts.

We generally use the "rule of thirds" when specifying the HARP imaging parameters. If the tag frequency is $\omega_{0}$ then the size of box on each side should also be $\omega_{0}$. This rule splits up the frequency space into 3 boxes of equal sizes: 2 around the harmonic peaks and 1 around the DC peak. With these parameters it is straightforward to show that $\pm 50 \%$ strain and the maximum rotational angle of $18^{\circ}-46^{\circ}$ degrees can be successfully imaged.



(a)



(c)



(b)

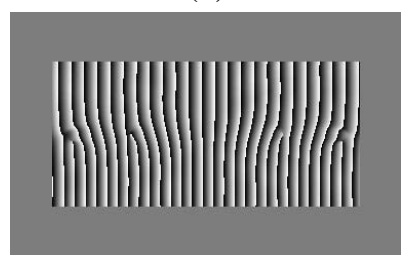

(d)
Fig. 4: (a) Tagged image (b) Feasible regions in space domain: white regions indicate regions where IF inside the bandpass filter. (c) Reconstructed tagged image; note the blurring in regions where the tags bend a lot (d) Phase estimate; Note the phase cuts in regions corresponding to regions of blurred tags.

Computer Simulated Phantom A computer simulation is shown in Fig. 4. Panel (a) shows three underlying tissue regions. The top region is under a horizontal strain of $-10 \%$ and the bottom region is under a horizontal strain of $-35 \%$. In the middle region, the strain varies in a cosine fashion, yielding a continuous bending of tags. The tagging and imaging parameters are the same as in the previous section. Panel (b) shows the set of points (white) whose IF is contained within the Fourier acquisition box implied by these parameters. Panel (c) shows the reconstructed tags after bandpass filtering; there is significant loss of tag fidelity in object regions that are black in Panel (b). In fact, misalignments of the tags in those regions, faintly apparent in Panel (c), are quite evident in the harmonic phase map shown in Panel (d). HARP tracking and Eulerian strain computations are erroneous in those regions as a result.

We can use Figure 3 to gain an intuitive appreciation of the results in Fig. 4. All object strains in this simulation lie in the range $-35--10 \%$, the range indicated by the vertical dash-dot lines in Fig. 3. The feasible domain in Fig. 3 indicates that the largest rotations that will be visible given this range of strains are in the range $18^{\circ}-22^{\circ}$, depending on the amount of strain. Those IFs in Fig. 4(a) disappearing after bandpass filtering are precisely those that have rotated beyond this visible range.

Cardiac MR data Figs. 5(a)-(d) demonstrate the validity of this theory on real cardiac MR image during mid-systole. All images in Fig. 5 have been cropped and masked for better visualization of the myocardium. Fig. 5(a) is a reconstructed tagged image, after applying a bandpass filter of 


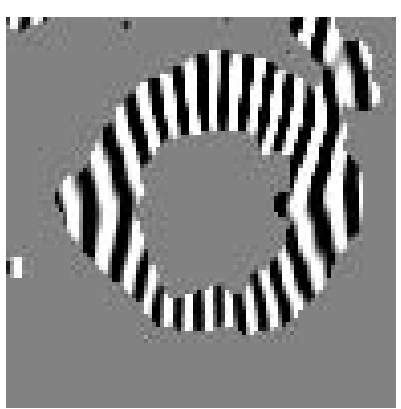

(a)

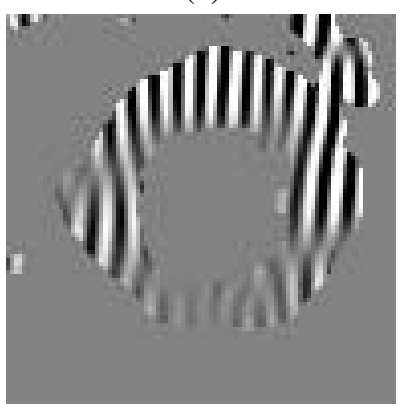

(c)

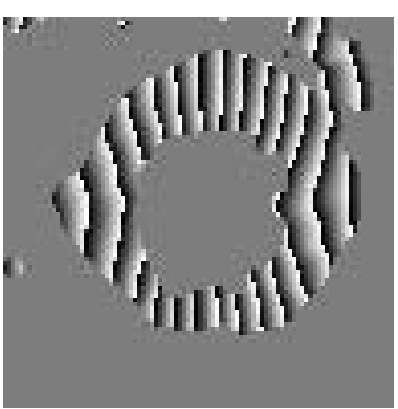

(b)

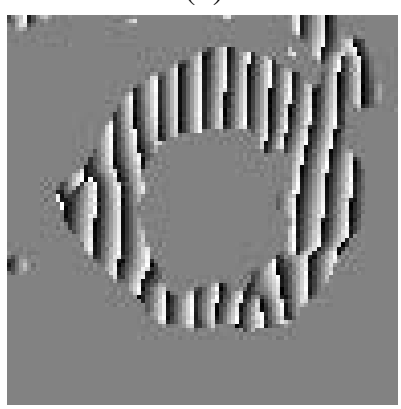

(d)
Fig. 5: (a) Reconstructed tags with $\mathrm{N}=33$. (b) Phase estimate with $\mathrm{N}=33$. (c) Reconstructed tags with $\mathrm{N}=15$; Note the loss in contrast in region where the tags bend or compress a lot (d) Phase estimate with $\mathrm{N}=15$; Note in regions of blurred tags the estimate of phase is inaccurate.

size 33. The original (uncropped) tagged image is shown in Fig. 1(a). From Figs. 5(a) and (b) we see that for bandpass filter size of 33 , both the tags and the phase are faithfully reproduced. Figs. 5(c) and (d) shows the effect of reducing the filter size to 15 . We see that in both regions of steep bending of tags and high radial expansion the tags get blurred out and corresponding phase estimates are inaccurate.

\section{CONCLUSION}

In this paper we provided a criterion for the selection of imaging and tagging parameters in order to use HARP successfully. Using an approximate relation between instantaneous and Fourier frequencies, we derived simple expressions for the dynamic range of elongation, compression, and rotation of tags. We concluded that the relatively small bandpass filter commonly used in current HARP applications is adequate for capturing the strains and rotations for both normal and diseased tissue. In the future, we will use this criterion to evaluate HARP in imaging tongue motion and extend this framework to characterize HARP resolution.

\section{References}

[1] N. F. Osman and J. L. Prince, "Visualizing myocardial function using HARP MRI," Phys. Med. Biol., vol. 45, no. 6, pp. 1665-1682, June 2000

[2] S. Sampath, A. Derbyshire, N. F.Osman, E. Atalar, and J. L. Prince, "Real-time imaging of two-dimensional cardiac strain using a fast harp pulse sequence," Magn. Res. Med., vol. 50, no. 1, pp. 154-63, July 2003.

[3] J.Garot, D. A. Bluemke, N. F. Osman, C. E. Rochitte, B. L. Gerber, J. L. Prince, and J. A. Lima, "Detailed analysis of transmural lv function and recruitable myocardial deformation in subendocardial vs transmural infarcts using taggedmri," Circulation, vol. 102, no. 18, Oct. 2000.

[4] J. Garot, D. A. Bluemke, N. F. Osman, C. E. Rochitte, E. A. Zerhouni, J. L. Prince, and J. A. Lima, "Transmural contractile reserve after reperfused myocardial infarction in dogs," J. Amer. Coll. Cardiol., vol. 36, no. 7, 2000.

[5] Kraitchman DL, Sampath S, Castillo E, Derbyshire J, Boston RC, Bluemke DA, Gerber BL, Prince JL, and Osman NF, "Quantitative ischemia detection during cardiac magnetic resonance stress testing by use of fastharp," Circulation, vol. 107, no. 15, pp. 2025-30, Apr. 2003.

[6] V. Parthasarathy, M. NessAiver, J. L. Prince, and M. Stone, "Tracking tongue motion from tagged magnetic resonance images using harmonic phase imaging (harp-mri)," in Proceedings of the 15th International Congress of Phonetic Sciences, Aug. 2003.

[7] P. J. Loughlin and B. Tacer, "Instantaneous frequency and the conditional mean frequency of a signal," Signal Processing, vol. 60, pp. 153-162, 1997.

[8] M. NessAiver and J.L.Prince, "Magnitude image cspamm reconstruction (micsr)," Magnetic Resonance in Medicine, vol. 50, no. 2, pp. 331-42, Aug. 2003.

[9] W. S. Kerwin and J. L. Prince, "A k-space analysis of MR tagging,” J. Magn. Res., vol. 142, pp. 313-322, 2000.

[10] B. Boashash, "Estimating and interpreting the instantaneous frequency of a signal - part 1: Fundamentals," Proceedings of the IEEE, vol. 80, no. 4, pp. 520-538, April 1992.

[11] B. Boashash, "Estimating and interpreting the instantaneous frequency of a signal - part 2: Algorithms and applications," Proceedings of the IEEE, vol. 80, no. 4, pp. 540-568, April 1992.

[12] L. Mandel, "Interpretation of instantaneous frequencies," American Journal of Physics, vol. 42, no. 10, pp. 840-846, Oct. 1974.

[13] D. Wei and A.C. Bovik, "On the instantaneous frequencies of multicomponent am-fm signals," IEEE Signal Processing Letters, vol. 5, no. 4, pp. 84-86, April 1998.

[14] P. M. Oliveira and V. Barroso, "Instantaneous frequency of multicomponent signal," Signal Processing, vol. 6, no. 4, pp. 81-83, April 1999.

[15] L. Cohen, Time-Frequency Analysis, Prentice Hall Signal Processing Series, 1995.

[16] N. F. Osman, Measuring Regional Cardiac Function using Harmonic Phase Magnetic Resonance Imaging, Electrical and computer engineering, Johns Hopkins University, Baltimore, MD, Feb. 2000. 